Fournal of Medical Genetics (1976). 13, 200-207.

\title{
Immunogenetic factors in aetiology of pre-eclampsia/eclampsia (gestosis)
}

\author{
J. S. SCOTT and D. M. JENKINS
}

From the Department of Obstetrics and Gynaecology, University of Leeds, 17 Springfield Mount, Leeds, LS2 9NG

Summary. The evidence that genetic and immunogenetic influences operate in the causation of pre-eclampsia/eclampsia (gestosis) is reviewed. The problems of definitive diagnosis are discussed along with the possibility of a multifactorial aetiology. The difficulties of differentiating trigger and effector mechanisms are also considered. It is concluded that there is evidence for a predisposition, probably genetic, operating in some cases, an immunogenetic mechanism in others, and chromosomal factors in a small group.

Early in this century it was the practice to group together a number of ill-understood maternal disorders occurring with pregnancy under the embracing title of 'toxaemia of pregnancy'. The term covered our ignorance of these widely differing states and it was positively misleading with its implication that a toxin existed when there was not a shred of evidence for this. The constituents of the group have now been differentiated into such conditions as hyperemesis gravidarium, hepatic necrosis from various causes, eclampsia/pre-eclampsia, cerebral haemorrhage, and thrombosis. All of these are now recognized as separate entities and their pathological mechanisms to a greater or lesser extent understood. The only conditions now left in the 'toxaemia' category are pre-eclampsia and eclampsia. These will be referred to here by the European term 'gestosis' which covers both and avoids the unjustified inference of the existence of a toxin.

Hippocrates noted that drowsiness, fits, and coma were of serious prognostic importance in pregnant women, and Varandaeus in 1619 used the term 'eclampsia' from the Greek because of the flashing lights often complained of before a fit. In 1843 Lever at Guy's Hospital reported that eclamptics had albuminuria, and subsequently with the appre-

Received 8 July 1975. ciation that hypertension and sometimes gross oedema were also present for considerable periods before fits occurred, the syndrome of 'pre-eclampsia' was identified.

It is important to appreciate that there is no precise diagnostic feature of the condition with the possible exception of multiple organ histology such as is usually only possible at necropsy. Not all convulsions in pregnancy are eclamptic and not all pregnancy hypertension is pre-eclamptic. Gestosis is frequently diagnosed by clinicians in late pregnancy when there is a minor rise in blood pressure-this is a wise and safe policy for mother and baby. However, many of these women do not have 'true' pre-eclampsia, and to include them in a study of aetiological factors adds a major element of confusion. It is unfortunately the case that where modern sophisticated facilities for investigative projects are available, the number of cases may be few and sometimes the response has been to lower the diagnostic standard. This is disastrous and problems of low diagnostic criteria almost certainly underly many of the contradictions which are to be found in published studies. It is, therefore, vital when considering literature reports to pay particular attention to the diagnostic criteria adopted in each paper. In studies on the aetiology of gestosis the diagnostic discrimination from other types of pregnancy hypertension must be high. Another aspect 200 
of the problem is that despite our lack of understanding of the aetiology and lack of any specific prophylactic or therapeutic measure, gestosis has become less frequent, less severe, and less fatal. This makes organized study more difficult.

Pathologically the main features are the presence of microthrombi and renal lesions with swelling of the glomerular capillary endothelial cells and amorphous fibrinoid deposition within the cells and beneath the basement membrane. Similar deposition is found in periportal tissues and in maternal vessels of the placental bed. Much recent evidence points to gross disturbance of the coagulation-fibrinolytic system balance with gestosis of the severe form. McKay et al (1953) have compared eclampsia with the Schwartzman reaction, an intravascular coagulation phenomenon demonstrable in animals, which apparently has an immunological basis. In pregnancy some factor alters the situation whereby the Schwartzman reation can be produced by a primary antigenic challenge while in the non-pregnant a secondary challenge is necessary. However, there is no agreed explanation as to the mechanism of initiation of this disorder. It is here the aetiological mystery currently rests.

Although the frequency and risks of gestosis have been reduced by modern care of a non-specific nature, it still remains a major cause of maternal and perinatal mortality demanding intensive study. In 1967-69 there were $73(16 \%)$ maternal deaths in England and Wales attributed to it, or in which it probably played a part, out of a total of 455 deaths assessed (Confidential Enquiries into Maternal Deaths in England and Wales, 1972). Furthermore when gestosis is severe there is a fourfold increase in perinatal mortality (Butler and Bonham, 1963).

In recent years many diseases of previously unknown aetiology have been shown to have an immunogenetic basis so it is appropriate to give careful consideration to this possibility in any condition which is still unexplained. This, of course, is particularly so with any pregnancy disease in which fetal-maternal immunogenic interaction may be involved.

In considering this possibility in gestosis, it may be helpful to bear in mind the words which Knox (1970) wrote when discussing the possibility of such an interaction between two individuals being responsible for anencephaly-'The laboratory demonstration of the immunological facts of rhesus haemolytic disease explained the population data with precision. This contrasts sharply with the failure of population studies to infer the immunological causes. Yet the relationship between the two is so exact that had the aetiological model been conceived before it was demonstrated, the popula- tion data were sufficient to declare it to be valid. This example is a salutary reminder to epidemiologists and population geneticists that there may be other diseases based upon genetic interactions whose aetiology is capable of being validated now if only the cipher could be solved.'

\section{General immunogenetic considerations}

Immunological factors may operate in the aetiology and they may also influence the severity of the established disease or may themselves be altered by the disease process. A particular immunogenetic pathology may be associated with a high incidence of gestosis but not all cases sharing that pathology carry the raised predisposition to gestosis. For example, haemolytic disease of the fetus due to Rhesus isoimmunization is a precisely defined immunogenetic process. In a small proportion of severe cases, fetal congestive cardiac failure (hydrops fetalis) develops. If these hydropic fetuses are allowed to languish in utero, which used to be the case, then two-thirds of the mothers develop gestosis (Scott, 1958). This high incidence does not apply to cases of Rhesus sensitization in general. Furthermore, hydrops fetalis may be caused by non-immunogenetic pathology, such as the haemoglobinopathies, cardiac defects, and infections. Hydrops fetalis in these cases is associated with a similar high incidence of gestosis. Thus, different mechanisms, immunogenetic and non-immunogenetic, may give rise to a similar gross pathology which is associated with a very high incidence of gestosis. Hydrops fetalis is responsible for only a very small percentage of cases of gestosis, but its association is important because it illustrates the necessity to allow the possibility of mixed immunogenetic and non-immunogenetic factors in aetiology. An immunogenetic factor may have a different role in different groups of patients such as primigravidae and multiparae. The fact that the incidence of gestosis in women pregnant for the second time in the same marriage (Platt, Stewart, and Emery, 1958) is almost half that in primigravidae seems to indicate that a previous pregnancy alters a woman's response or that some other variable has been introduced that modulates the development of the disease process. Again, immunogenetic mechanisms may be involved at one, or more than one stage of the disease process, for example, at a primary (or induction) phase, or at a secondary (or effector) phase, or both.

\section{Genetic data}

Two lines of evidence of a genetic basis for gestosis may be considered. One involves the idea that a woman is genetically coded for a high gestosis risk; 
the other relates to the possibility that some interaction between the fetus and the mother is the result of an inherited factor in one or the other.

(1) Theoretical models. Several studies have been performed based on theoretical immunogenetic models of gestosis but in most, probably incorrectly, it has been assumed that a single genetic factor is involved. Penrose (1946) considered a simple ' $a / a$ ' mother and ' $a / A$ ' fetus situation analogous to the Rhesus system. ' $A$ ' in this theory is the offending antigen that causes an immune reaction leading to gestosis. If this simple model were correct neither the pregnancy from which an affected mother was herself born nor any of the daughters of an affected pregnancy could have the condition, but as Chesley's data (see below) show this is far from being the case. Kalmus (1946) independently considered a similar mechanism to that postulated by Penrose but pointed out that the disease aetiology might be based on a more complex genetic situation. Platt et al (1958) considered a proposition essentially the reverse of that of Penrose and Kalmus. They postulated that gene ' $E$ ' responsible for the disease was carried by the mother as 'e/E'. An 'e/e' fetus might react to the maternal ' $E$ ' antigen producing an antibody which was reabsorbed into the maternal circulation affecting maternal cells. As with Penrose's model, neither the mothers nor the daughters of affected patients could have the disease. In Platt's study no mother of a patient with gestosis had shown gestosis in the pregnancy resulting in the patient's birth, though 3 out of 54 mothers of patients in this category had experienced gestosis in other pregnancies. Among another 100 women who had gestosis, 3 of their own mothers had gestosis in the pregnancy bearing the patient, thus providing evidence against the proposition in its simplest form. They did, however, regard their data as giving some support to their postulate, in that there was an increased incidence of gestosis in sisters, brother's wives, husband's sisters, and husband's brothers' wives, than in mothers of patients who had gestosis.

The models of Penrose and Platt involve the presumption of antibody production. Alternative immunogenetic mechanisms are theoretically possible including induction of immune tolerance. Maternal non-response to fetal antigenic challenge, as distinct from induced tolerance, is also a possible mechanism. There is growing evidence that immunocompetence may be genetically controlled (Katz, 1975), and it may follow that diseases resulting from some immune mechanisms are hereditary.
(2) Family studies. Chesley, Annitto, and Cosgrove (1968) have marshalled the evidence concerning a familial tendency in gestosis. Much of the older literature is ancecdotal and of doubtful authenticity, but in Chesley's own careful study of 260 surviving eclamptics from the years 1931 to 1951, he showed an incidence of eclampsia in the daughters of these women eight times higher than in indigent controls. The incidence of nonconvulsive gestosis was also high and it was clear that the daughters of women who have had gestosis not only may suffer from the condition but have an increased incidence. Grand-daughters of gestosis patients also had a high incidence. By contrast the incidence of gestosis in daughters-in-law was less than one-third that in daughters and close to that in the general population. This is strong suggestive evidence that gestosis 'runs in families', though Chesley allowed alternative explanations relating to environment. Adams and Finlayson (1961) studied the sisters of 146 primigravidae who developed preeclampsia or some degree of hypertension in pregnancy. They showed a higher frequency of preeclampsia or hypertension in their first pregnancies compared with the sisters of 273 primigravidae without pre-eclampsia or hypertension, and the authors concluded that their findings suggested a strong familial tendency in these conditions.

(3) Population studies. Stevenson et al (1971) in a study in Ankara, where there is a relatively high incidence of consanguinity, found a lower incidence of gestosis in pregnancies where mother and father were related, than in a local population. This difference derived mainly from cases of mild gestosis in which the precision of diagnosis is lower. The authors pointed out that on the Penrose/Kalmus hypothesis the incidence of gestosis would always be less when women were related to their husbands regardless of the gene frequency while on the Platt hypothesis the incidence would be less if the gene frequency was $<0.5$ These considerations refer to a simple single locus postulate which seems on present evidence unlikely to be correct.

One of the undisputed facts about gestosis is that it occurs more frequently in multiple than in singleton pregnancy. If gestosis relates to the presence of an antigen either in the fetus or mother which is foreign to the other, then the chance of such disparity in dizygous twins would be up to double that in monozygous twins or singleton gestations. In four different twin populations studied by Stevenson et al (1971) gestosis was commoner in unlikesex (definitely dizygous) than in like-six twin pregnancies. The differences were of borderline 
significance but applying Weinberg's hypothesis to provide data for 'estimated' monozygous twins, significance at the 5 per cent level was obtained. McFarlane and Scott (1976) in a similar study in a larger series in one hospital did not find any difference between the incidence of gestosis in unlike- and like-sex twins. Using Weinberg's hypothesis estimates were made of presumed monozygous twins and only in multiparae was there a significantly lower figure for gestosis in 'estimated' monozygous cases. In primigravidae the figure was reversed and when both parity groups were considered together there was no significant difference. In interpreting these data it is relevant that in multigravidae the order of accuracy of diagnosis of gestosis is much lower than in primigravidae. A comparison of twins with matched singleton pregnancies showed that the incidence of gestosis with twins was three times that with singletons, and many studies have reported figures of this order or greater. If the disease-inducing antigen is fetal, it is possible that if the twins are monozygous there will be a double dose of antigen and the gestosis will be of greater severity. If the twins are dizygous and the father a heterozygote for the inducingantigen there will be at most a double chance that at least one conceptus will carry the antigen and, therefore, a double chance of gestosis developing. If this were the explanation of the increased incidence of gestosis in twins the order would be $<\frac{2}{3} \times 2$ (approximately $\frac{2}{3}$ of twins being dizygous), whereas the observed increase, as stated, is of the order of 3 or greater. This suggests that the factors influencing the increased incidence of gestosis in multiple pregnancy are not simply additive relating to the number of fetuses present.

\section{Sex ratio}

It has been suggested that gestosis is commoner in pregnancies associated with a male fetus (Salzmann, 1955; Toivanen and Hirvonen, 1970). Toivanen and Hirvonen found that the sex ratio was increased with the severity of the gestosis. They believed that this phenomenon could be explained on the basis of potentiation of histocompatibility antigens carried on the $\mathrm{Y}$ chromosome and quoted as evidence the higher incidence of HL-A antibodies found in women with gestosis compared with healthy parturients (Tiilikainen, 1971). More recent evidence shows no increased incidence of HL-A antibodies in gestosis (C. Redman, 1975, personal communication, 1976; J. Need, D. M. Jenkins, and J. S. Scott, 1975, unpublished data). Johansen and Festenstein (1974) have reported that a male fetus evokes a stronger response than a female in terms of HL-A antibody production, particularly in primigravidae. They suggested that this might be attributed to the presence of a sex-linked histocompatibility system in man which reacts with the HL-A system. A fetal antigen responsible for gestosis linked to this system might explain the reported increased sex ratio in gestosis. However, other evidence suggests that the multiparous female is, if anything, less responsive to male associated antigens than to female antigens; Oliver (1974), in an analysis of 349 cadaver renal grafts, has shown significantly better survival of female recipients of male as opposed to female grafts. Survival of male recipients was intermediate between the two extremes of female recipients and no influence of donor sex was apparent. Brochier, Roitt, and Festenstein (1974) have shown that certain sera from multiparous women prevent mixed leucocyte culture response of female lymphocytes when they are stimulated by male HL-A incompatible lymphocytes. McFarlane and Scott (1976) showed no significant difference in the incidence of gestosis in twin pregnancies in which there was a male fetus compared with those in which both twins were female.

\section{Triploidy}

Much information has now accumulated on pregnancies in which there are chromosomal abnormalities in the conceptus. In one category onlytriploidy-there seems to be a major predisposition for the pregnancy to be accompanied by gestosis and for that to present at a remarkably early stage of gestation. Paterson et al (1971) reported 2 cases of $69 \mathrm{XXX}$ triploidy associated with severe gestosis developing at 25 and 29 weeks, respectively. Toaff and Peyser (1974) reviewed 14 cases of triploidy in which information was available and 8 had severe gestosis, generally at 16 to 24 weeks' gestation-a stage of pregnancy at which gestosis is extremely uncommon. In all the cases with gestosis the placenta was large, the placental coefficient high (mean 0.83 ; normal 0.2 ) and areas of molar degeneration were present. They regarded the mechanism through which triploidy induced gestosis as that of 'hyperplacentosis' described by Scott (1958).

A striking feature in these cases is that though the trophoblastic mass is increased it is not enormous when considered against term pregnancies and certainly much less than in hydrops fetalis, hydatidiform mole, diabetes, and twins-all regarded as typical examples of hyperplacentosis. This suggests that qualitative factors may be involved. For each nucleus, of course, the triploid trophoblast will 
have 50 per cent more chromosomal material than a normal diploid nucleus. Furthermore, triploid conceptuses may be a consequence of dispermy (Polani, 1969), in which case the degree of conceptus-maternal antigenic incompatibility would be much greater than normal. The discovery of this association of gestosis with triploidy is one of the few new correlations to emerge and further study of it offers promise in helping to clarify the aetiological mystery.

\section{HL-A system}

The mutual tolerance of incompatible tissues by mother and fetus is the major unexplained phenomenon of pregnancy physiology; gestosis is the major unexplained phenomenon of pregnancy. It is, therefore, a possibility that an understanding of the factors concerned with tissue incompatibility may help to explain the gestosis puzzle.

The inheritance of immune response genes which confer an ability to respond to a particular antigenic challenge in a manner which is not possible in their absence has obvious implications in the context of fetal-maternal immunogenetic reactions. For example, if gestosis is the result of a fetalmaternal immunogenetic interaction consequent on either mother or fetus bearing a 'gestosis-inducingantigen', then whether or not gestosis occurs in such a pregnancy may depend upon whether the individual challenged is capable of a response or not. Either the positive or negative situation may be important. In parous women the incidence of antiHL-A antibodies is about $30 \%$ while even in primigravida figures of $12 \%$ have been reported (Tiilikainen, 1971). These antibodies are specific for the HL-A antigens carried on fetal tissue which are inherited from the father. Exactly how these fetal HL-A antigens induce maternal antibody production is not clear. If it were established that trophoblast exhibited HL-A antigens then this would be an obvious explanation, but this is not yet clear. It seems likely that fetal lymphocytes circulate in the maternal circulation for prolonged periods after delivery (Tiilikain $\in \mathbf{n}$, Schroder, and de la Chapelle, 1974) but their very survival in the immunogenetically competent host may depend on the non-expression or masking of paternally derived HL-A antigens on their surface. Papiernik and Lespes (1973) have shown that patients with obstetric complications associated with fetal-maternal transfusion have a greatly increased incidence of anti-HL-A antibodies. It is also possible that HL-A antigens in some soluble form or attached to some carrier may pass from fetus to mother and induce antibody formation.
Terasaki et al (1970) stressed that in spite of the fact that $99 \%$ of fetuses are HL-A incompatible with their mothers, only $50 \%$ of multiparous women produce lymphocytotoxins. The women who do not produce lymphocytotoxins may be immunogenetic non-responders analogous to the $50 \%$ recipients of multiple transfusions who do not form lymphocytoxins or the $50 \%$ kidney transplant recipients who do not reject grafts despite histocompatibility differences.

Very little is known about the HL-A system and pregnancy phenomena. Jenkins and Good (1972) and Jenkins (1973) suggested from a preliminary study that there was a weak association between maternal-paternal HL-A disparity and increased placental weight in uncomplicated pregnancies. Carretti et al (1974) found lymphocytotoxic antibodies more frequently in patients with gestosis than in normal pregnant women. Fingleton (1971), however, reported a retrospective study of 78 women whose sera had been shown to contain leucocytotoxic antibodies during pregnancy; the incidence of gestosis in this group was compared with the incidence in a control group without antibodies and no significant difference was shown. Unpublished prospective studies of severe gestosis in Leeds have not shown an increased incidence of lymphocytotoxic antibodies in gestosis.

Tiilikainen's study (1971) revealed no obvious correlation between the child's paternal HL-A haplotype and the maternal toxaemia. 'Harmless' sibs with the same haplotype had been born before and after the 'noxious' ones. Leucocyte antigens in this study were not subjected to detailed analysis, but no major differences were found between families in which the mother suffered gestosis and controls. Two mothers suffering gestosis are described: they developed lymphocytotoxic antibodies, but the relevant antigen was inherited by all their children not only those associated with gestosis.

It is possible that in some circumstances such antibodies might be removed from the circulation as antigen-antibody complexes in kidney or placenta as quickly as they are formed. There are at least superficial similarities between some lesions of gestosis and those of glomerulonephritis which are generally attributed to antigen-antibody complexes. It would be interesting to know the relative amount of elutable anti-HL-A antibody in placentas from normal pregnancies and pregnancies complicated by gestosis. There is suggestive evidence that homozygosity at the HL-A loci may predispose to gestosis (C. Redman, 1975, personal communication). Hypothetical autoimmune mechanisms in 
vascular disease and gestosis have been discussed by Mathews, Whittingham, and Mackay (1974).

\section{Blood group antigens}

Dienst's (1905) postulate of a simple blood group incompatibility between mother and fetus as an aetiological factor in gestosis was the first of many such theories. McQuarrie (1923) discussed a possible relation between 'interagglutination' and gestosis on the basis of the observation of agglutinated red cells in the hyaline thrombi found in the characteristic areas of necrosis in the liver of women dying from eclampsia. Gestosis appeared to be 16 times more likely to occur when the maternal and fetal bloods were incompatible than when they were of the same blood group. Pike and Dickens (1954) in a study of 3651 pregnant women reported that patients with gestosis showed a significant excess of blood group $\mathrm{O}$ over patients with normal pregnancies but they were unable to confirm this finding in a second study reported two years later. Pearson and Pinker (1956) and Andrews (1959) also failed to find any evidence of an association between gestosis and $\mathrm{ABO}$ or Rhesus blood group distribution, though Chandravati (1966) showed a higher frequency of group $\mathrm{O}$ mothers, associated with a higher frequency of $\mathrm{ABO}$ heterospecific pregnancies in gestosis. May (1973), on the other hand, reported a preponderance of blood group A among patients with gestosis. It has also been shown that group A subjects tend to be more at risk of venous thromboembolic disease associated with pregnancy and oral contraception, and this is of interest as gestosis is also associated with intravascular coagulation. Jick et al (1969) and Harlap and Davies (1974) were unable to find an excess of gestosis among group A mothers, but reported an apparent interaction between $\mathrm{ABO}$ and Rhesus blood group, with $\mathrm{O}$ Rhesus positive mothers having fewer and $O$ Rhesus negative mothers more cases of gestosis than expected. They warned that since the incidence of gestosis might be influenced by many variables which have a relation with one or other blood group then these variables must be considered in assessing diseaseblood group relations.

\section{Placental studies}

Not only are certain obstetric conditions with large placental mass associated with a high incidence of gestosis (Scott, 1958), but in gestosis it has been reported that perhaps 20 times more trophoblast may be found in the uterine veins than in normal pregnancies (Jaameri, Koivuniemi, and Carpen, 1965).
This may be regarded as representing an increased immune challenge to the maternal host. Loke, Joysey, and Borland (1971) and Taylor and Hancock (1975) have indicated the likely presence of HL-A antigens on late gestation trophoblast, albeit in low density. No studies have been done to assess whether there is increased antigen density or immunogenicity of trophoblast in gestosis, though there is a theoretical basis for doing so in view of the fact that fetal triploidy is associated with a strong tendency to gestosis.

Svetoslavova et al (1973) have reported the presence of trophoblast antibodies in serum in both gestosis and normal pregnancy. Using the antiglobulin consumption test, the level of trophoblast antibodies in the sera of women with clinical 'toxaemia' was compared with that in normal primigravidae and normal multigravidae using nonpregnant controls. These antibodies were most prevalent in 'toxaemia' patients, but this group included a majority of cases labelled 'nephritis of pregnancy'. Irino, Okuda, and Grollman (1967) were able to induce a renal lesion in rats by injecting isologous placental extracts in Freund's complete adjuvant. These changes were comparable to those observed in human gestosis and were associated with albuminuria and hypertension; they were considered to be the result of an antigen-antibody reaction.

In view of the associated high incidence of gestosis with hydatidiform mole, the question of the antigenicity of molar tissue is of interest. Lawler (1974) reported that paternal specific anti HL-A antibodies may be detected following first pregnancies involving hydatidiform mole. It does not follow, however, that the antigens involved must have arisen from the abnormal chorionic tissue since embryonic structures may have been present at a very early stage and could have given rise to these antigens. Like hydatidiform mole, twin pregnancies and hydrops fetalis are associated with an increased mass of placental tissue (hyperplacentosis) and an increased incidence of early gestosis.

\section{Cell-mediated immune response}

Evidence is accumulating concerning the in vitro response of small lymphocytes to different mitogens and antigens in normal pregnancy and gestosis. In normal pregnancy immune reactivity is reduced with respect to specific paternal antigen stimulation (Jenkins and Hancock, 1972) and to non-specific cellular (Jones and Curzen, 1973) and non-cellular antigens (Hill, Finn, and Denye, 1973). Maternal plasma has been shown by many workers (Jones, Curzen, and Gaugas, 1973; Kasakura, 1974) and 
others to have immunosuppressive effects one cellular responses 'in vitro'. This effect is seen to increase in late pregnancy and with increasing parity; it may have specific and non-specific immunosuppressive components. Using 'one-way' mixed lymphocyte reactions in a family with 24 children, Tiilikainen (1971) reported finding the maternal response to 'noxious' children usually either faster or higher than the response to 'harmless' children, both of whom had inherited the same paternal haplotype.

In gestosis the maternal lymphocyte response to PHA has been found to be reduced (J. Need and D. M. Jenkins, 1976, unpublished data) but not to paternal cellular antigens (Curzen, Gaugas, and Jones, 1973). There is no evidence that maternal plasma in gestosis is more or less immunosuppressive, than in normal pregnancy. Byles (1974) recorded a significantly raised level of spontaneous transformation in washed lymphocytes from mothers with gestosis as compared with normal controls. This may represent an altered population of lymphocytes in gestosis.

Need (1975) recorded a patient who at 20 years of age had twins in her first pregnancy by her first husband and this was uncomplicated by gestosis. Her second pregnancy at 24 years was by a second husband and was complicated by very severe gestosis, with the baby dying in the neonatal period. The chances of this occurring by chance are extremely small and it was considered possible that some differences in the immunogenetic relation between the mother and the two fathers might be responsible. Studies were done on blood groups and HL-A types of both fathers and mother, and mixed lymphocyte reactions (MLR) were performed between them. There was a lower one-way MLR between father 1 (stimulator) and the mother as compared with father 2 and the mother. Father 1 had no HL-A antigens not carried by the mother. While it is impossible to draw conclusions from such a single study, the design of the investigations could serve as a model for other studies in similar unusual 'family' situations when they are encountered. It is possible that a small number of studies on such lines will lead to more information on the paternal contribution to gestosis than very much larger studies based on single father-mother combinations.

\section{Conclusion}

The existing knowledge concerning the role of immunogenetic factors in gestosis is fragmentary. Confusion may arise because gestosis, as clinically defined, may not be a single disease entity. More than one pathological mechanism and aetiological trigger may be involved and genetic or immunogenetic factors may be operative in some but not all cases. It is clear, however, that gestosis carries an inherited predisposition to its occurrence and in a few cases the primary trigger is immunogenetic (Rhesus hydrops) and in others chromosomal (triploidy). Immunological phenomena, e.g. immune complexes are becoming more amenable to study, and it may be that interrelation of the complex changes in different systems-genetic, immunological, endocrine, coagulation, vasopressor, etc.seen in gestosis may soon become clearer.

\section{REFERENCES}

Adams, A. E. and Finlayson, A. (1961). Familial aspects of preeclampsia and hypertension in pregnancy. Lancet, 2, 1375-1378. Andrews, G. S. (1959). Blood groups and toxaemia of pregnancy. British Medical fournal, 2, 806-807.

Brochier, J. P., Roitt, I., and Festenstein, H. (1974). Inhibition of lymphocyte proliferative responses by anti-HL-A alloantisera. European fournal of Immunology, 4, 709-714.

Butler, N. R. and Bonham, D. G. (1963). Perinatal Mortality. The First Report of the 1958 British Perinatal Mortality Survey. Livingstone, Edinburgh and London.

Byles, A. B. (1974). 7th Meeting of the Organisation Gestosis, TelAviv, October.

Carretti, N., Fagiolo, U., Zanetti, M., and Chiaramonte, P. (1974). Association of anti-HL-A antibodies with toxaemia in pregnancy. In Immunology in Obstetrics and Gynaecology, pp. 221-225. Excerpta Medica, Amsterdam.

Chandravati, Das (1966). M.D. Thesis, King George Medical College, Lucknow, India.

Chesley, L. C., Annitto, J. E., and Cosgrove, R. A. (1968). The familial factor in toxaemia of pregnancy. Obstetrics and Gynecology, 32, 303-311.

Confidential Enquiries into Maternal Deaths in England and Wales 1967-1969 (1972). Her Majesty's Stationery Office, London.

Curzen, P., Gaugas, J. M., and Jones, E. (1973). In vitro studies to investigate feto-maternal immunological relationships in pregnancy. In 1st International Congress on Immunology in Obstetrics and Gynaecology, Padua, pp. 19-20. International Congress Series No. 281. Excerpta Medica, Amsterdam.

Dienst, A. (1905). Das Eklampsiegift. Zentralblatt für Gynäkologie, 29, 353-364.

Fingleton, A. M. (1971). Leucocytotoxic antibodies and preeclampsia of pregnancy. Transplantation, 12, 319-321.

Harlap, S. and Davies, A. M. (1974). Maternal blood group A and pre-eclampsia. British Medical fournal, 3, 171-172.

Hill, C. A., St., Finn, R., and Denye, V. (1973). Depression of cellular immunity in pregnancy due to a serum factor. British Medical fournal, 3, 513-514.

Irino, T., Okuda, T., and Grollman, A. (1967). Changes induced in the glomeruli of the kidney of rats by placental extracts as observed with the electron microscope. American fournal of Pathology, 50, 421-433.

Jaameri, K. E., Koivuniemi, A. P., and Carpen, E. O. (1965) Occurrence of trophoblasts in the blood of toxaemic patients. Gynaecologia, 160, 315-320.

Jenkins, D. M. (1973). Immunological studies of the lymphocyte in human pregnancy. M. D. Thesis, Queen's University, Belfast.

Jenkins, D. M. and Good, S. (1972). Mixed lymphocyte reaction and placentation. Nature New Biology, 240, 211-212.

Jenkins, D. M. and Hancock, K. W. (1972). Maternal unresponsiveness to paternal histocompatibility antigens in human pregnancy. Transplantation, 13, 618-619.

Jick, H., Westerholm, B., Vessey, M. P., Lewis, G. P., Stone, D., Inman, W. H., Shapiro, S., and Worcester, J. (1969). Venous thrombo-embolic disease and ABO blood type. Lancet, 1, 539545.

Johansen, K. and Festensein, H. (1974). Maternal HL-A antibodies and fetal sex. British Medical fournal, 4, 202-203. 
Jones, E. and Curzen, P. (1973). The immunological reactivity of maternal lymphocytes in pregnancy. Fournal of Obstetrics and Gynaecology of the British Commonwealth, 80, 608-610.

Jones, E., Curzen, P., and Gaugas, J. M. (1973). Suppressive activity of pregnancy plasma on the mixed lymphocyte reaction. Fournal of Obstetrics and Gynaecology of the British Commonwealth, 80, 603-607.

Kalmus, H. (1946). Genetic antigenic incompatibility as a possible cause of the toxaemia occurring late in pregnancy. Annals of Eugenics, 13, 146-149.

Kasakura, S. (1974). Suppressive activities of pregnancy and fetal plasmas on the reactivity of lymphocytes to various stimulants. In Immunology in Obstetrics and Gynaecology, pp. 134-146. Excerpta Medica, Amsterdam.

Katz, D. H. (1976). The role of histocompatibility gene products in immune recognition and cellular interactions. In 9th Leucocyte Culture Conference, Williamsburg, Virginia, December 1-5th, 1974. Academic Press, London. In the press.

Knox, E. G. (1970). Fetus-fetus interaction-a model aetiology for anencephaly. Developmental Medicine and Child Neurology, 12, 167-177.

Lawler, S. D. (1974). The significance of HL-A antibodies in trophoblast neoplasias. In Immunology in Obstetrics and Gynaecology, pp. 310-311. Excerpta Medica, Amsterdam.

Loke, Y. W., Joysey, U. C., and Borland, R. (1971). HL-A antigens on human trophoblast cells. Nature (London), 232, 403-404.

McFarlane, A., and Scott, J. S. (1976). Pre-eclampsia/eclampsia in twin pregnancies. Fournal of Medical Genetics, 13, 208-211.

McKay, D. G., Merrill, S. J., Weiner, A. E., Hertig, A. T., and Reid, D. E. (1953). Pathologic anatomy of eclampsia, bilateral rena cortical nesrosis, pituitary necrosis and other acute fatal complications of pregnancy and its possible relationship to generation Schwartzman phenomena. American fournal of Obstetrics and Gynaecology, 66, 507-539.

McQuarrie, I. (1923). A possible relationship between interagglutination and the toxaemias of pregnancy. Fohns Hopkins Hospital Bulletin, 34, 51-59.

Mathews, J. D., Whittingham, S., and MacKay, I. R. (1974). Autoimmune mechanisms in human vascular disease. Lancet, 2, 14231427.

May, D. (1973). Maternal blood group A and pre-eclampsia. British Medical fournal, 4, 738.

Need, J. (1975). Pre-eclampsia in pregnancy by different fathers: immunological studies. British Medical fournal, 1, 548-549.

Oliver, R. T. D. (1974). Are there Y-linked histocompatibility antigens in man? European fournal of Immunology, 4, 519-520.

Papiernik, E. and Lespes, S. (1973). Clinical selection and incidence of anti-HL-A antibodies in pregnant women. In 1st International Congress on Immunology in Obstetrics and Gynaecology, Abstract No.
60. International Congress Series, No. 281. Excerpta Medica, Amsterdam.

Paterson, W. G., Hobson, B. H., Smart, G. E., and Bain, A. D. (1971). Two cases of hydatidiform degeneration of the placenta with fetal abnormality and triploid chromosome constitution Journal of Obstetrics and Gynaecology of the British Commonwealth, 78, 136-142.

Pearson, M. G., and Pinker, G. D. (1956). ABO blood groups and toxaemia of pregnancy. British Medical fournal, 1, 777-778.

Penrose, L. S. (1946). On the familial appearances of maternal and fetal incompatibility. Annals of Eugenics, 13, 141-145.

Pike, L. H. and Dickens, A. M. (1954). ABO blood groups and toxaemia of pregnancy. British Medical fournal, 2, 321-323.

Platt, R., Stewart, A. E., and Emery, E. W. (1958). The aetiology incidence and heredity of pre-eclamptic toxaemia of pregnancy. Lancet, 1, 552-556.

Polani, P. E. (1969). Autosomal imblance and its syndromes, excluding Down's. British Medical Bulletin, 25, 81-93.

Salzmann, K. D. (1955). Do transplacental hormones cause eclampsia? Lancet, 2, 953-956.

Scott, J. S. (1958). Pregnancy toxaemia associated with hydrops fetalis, hydatidoform mole and hydramnios. Fournal of Obstetrics and Gynaecology of the British Empire, 65, 689-701.

Stevenson, A. C., Davison, B. C. C., Say, B., Ustuoplu, S., Durmas, L., Abdul-Einen, M., and Toppozada, H. K. (1971). Contribution of feto-maternal incompatibility to aetiology of pre-eclamptic toxaemia. Lancet, 2, 1286-1289.

Svetoslavova, E., Momchilor, B., Hristova, G., Enchev, E., and Yalamova, D. (1973). Immunological studies on taxaemia of pregnancy. In Immunology of Reproduction, pp. 418-425. Bulgarian Academic Sciences Press, Sofia.

Taylor, P. and Hancock, K. W. (1975). Antigenicity of trophoblast and some possible antigen-masking effects during pregnancy. Immunology, 28, 973-982.

Terasaki, P. I., Mickey, M. R., Yamazaki, J. N., and Vredevoe, D. (1970). Materno-fetal incompatibility. Transplantation, 9, 538543.

Tiilikainen, A. (1971). Feto-maternal histoincompatibility in toxaemia of pregnancy. In Human Anti-Human Gammaglobulins, pp. 223-227. Pergamon Press, Oxford and New York.

Tiilikainen, A., Schroder, J., and Chapelle, A de la (1974). Fetal leucocytes in the maternal circulation after delivery. Transplantation, 17, 355-360.

Toaff, R. and Peyser, M. R. (1974). 7th Meeting of the Organisation Gestosis, Tel-Aviv, October 1974.

Toivanen, P. and Hirvonen, T. (1970). Sex ratio of newborns: preponderance of males in toxaemia of pregnancy. Science, 170, 187188. 